\title{
Projecting Signed Two-mode Networks
}

DOI:

10.1080/0022250X.2019.1711376

\section{Document Version}

Accepted author manuscript

Link to publication record in Manchester Research Explorer

\section{Citation for published version (APA):}

Schoch, D. (2021). Projecting Signed Two-mode Networks. Journal of Mathematical Sociology, 45(1), 37-50. https://doi.org/10.1080/0022250X.2019.1711376

\section{Published in:}

Journal of Mathematical Sociology

\section{Citing this paper}

Please note that where the full-text provided on Manchester Research Explorer is the Author Accepted Manuscript or Proof version this may differ from the final Published version. If citing, it is advised that you check and use the publisher's definitive version.

\section{General rights}

Copyright and moral rights for the publications made accessible in the Research Explorer are retained by the authors and/or other copyright owners and it is a condition of accessing publications that users recognise and abide by the legal requirements associated with these rights.

\section{Takedown policy}

If you believe that this document breaches copyright please refer to the University of Manchester's Takedown Procedures [http://man.ac.uk/04Y6Bo] or contact uml.scholarlycommunications@manchester.ac.uk providing relevant details, so we can investigate your claim.

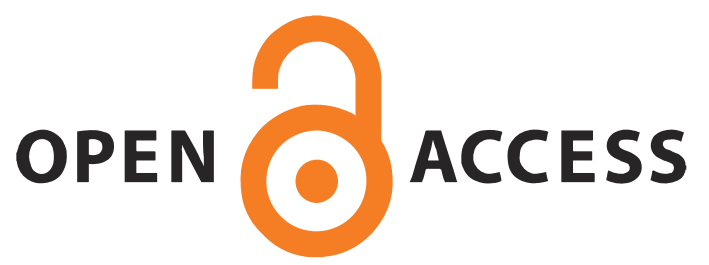




\title{
Projecting Signed Two-mode Networks
}

\author{
David Schoch ${ }^{1}$ \\ 1 The Mitchell Centre for Social Network Analysis, \\ Department of Sociology, \\ The University of Manchester \\ david.schoch@manchester.ac.uk \\ Forthcoming in Mathematical Sociology, 2020
}

\begin{abstract}
Signed two-mode networks have so far predominantly been analysed using blockmodeling techniques. In this work, we put forward the idea of projecting such networks onto its modes. Two projection methods are introduced which allow the application of known dichotomization tool for weighted networks to obtain a simple signed network. It turns out, however, that resulting networks may contain ambivalent ties, defined as conjunctions of positive and negative ties. We show that this requires the reformulation of matrices related to the network and introduce the complex adjacency and Laplacian matrix. These matrices are used to prove some properties related to balance theory including ambivalence.
\end{abstract}

Keywords: Signed Networks; Balance Theory; Projections

\section{Introduction}

Research on signed networks (that is, networks with both positive and negative ties) is mainly driven by balance theory (Heider, 1946), its generalized 
form by Cartwright and Harary (1956), and the concept of clusterability by (Davis, 1967). Many empirical studies exist that investigate the theory in different settings, including social media (Kunegis et al., 2009; Leskovec et al., 2010), Wikipedia (Brandes et al., 2009; Brandes and Lerner, 2008; Kittur et al., 2007) and international relations (Doreian and Mrvar, 2015; Estrada, 2019; Moore, 1979) using a plethora of measures to assess the "balancedness" of a network (Aref and Wilson, 2018; Estrada, 2019; Estrada and Benzi, 2014; Facchetti et al., 2011; Giscard et al., 2017; Kirkley et al., 2019; Ma et al., 2015, 2017; Marvel et al., 2009; Terzi and Winkler, 2011).

Comparably little attention has been paid to adapting other network analytic tasks such as, for instance, the derivation of centrality for signed networks. While there exist a variety of indices (Bonacich and Lloyd, 2004; Everett and Borgatti, 2014; Kamvar et al., 2003; Kerchove and Dooren, 2008; Traag et al., 2010; Wu et al., 2016), there seems to be no deeper understanding of the substantive foundation of the concept for signed networks. A more developed area of research is the adaption of blockmodeling for signed networks, put forward predominantly by Doreian and collaborators (Doreian, 2004, 2008; Doreian et al., 1996; Doreian and Mrvar, 1996, 2009, 2015). A more rigorous assessment of the applicability of general network analytic tools on signed networks is offered by Everett and Borgatti (2014).

In this work, we focus on signed two-mode networks and address the question of how to derive and analyse their one-mode projections. Signed two-mode networks have so far mostly been studied with blockmodeling techniques, ignoring the possibility of projections. Examples include studies on the voting behavior of justices on the U.S. Supreme Court (Doreian and Mrvar, 2009) or on roll-call votes in the United Nations General Assembly (Doreian et al., 2013). 
The main goal of this paper is to adapt known projection tools to signed networks and discuss emerging peculiarities, such as the presence of both a positive and negative tie between two nodes in the projection. This gives rise to ambivalent ties (Abelson and Rosenberg, 1958; Cartwright and Harary, 1970), a new type of tie which requires a re-formulation of adjacency and Laplacian matrices in terms of complex numbers. These matrices are then used to prove some properties related to balance theory in the presence of ambivalent ties.

\section{Terminology}

A signed one-mode network is an ordered pair $\Gamma=(G, \sigma)$, where $G=(V, E)$ is the underlying graph with vertex set $V$ and edge set $E \subseteq V \times V$, and $\sigma ; E \rightarrow\{+1,-1\}$ is a sign function. The cardinalities of $V$ and $E$ are given by $|V|=n$ and $|E|=m$, respectively. Throughout this work we consider all networks to be connected and undirected, i.e. the edge set consists of unordered pairs $\{j, k\}$, where $j, k \in V .{ }^{1}$

The signed adjacency matrix $A$ is an $n \times n$ matrix with entries $A_{j k}=$ $\sigma(\{j, k\})$ if $\{j, k\} \in E$ and $A_{j k}=0$ otherwise. The signed Laplacian matrix $\bar{L} \in \mathbb{R}^{n, n}$ is defined as $\bar{L}=\bar{D}-A$, where $\bar{D} \in \mathbb{R}^{n, n}$ is a diagonal matrix with entries $\bar{D}_{j j}=\sum_{k}\left|A_{j k}\right|$.

A signed two-mode network is an ordered pair $\Gamma=(G, \sigma)$, where $G=$ $(U, V, E)$ is the underlying two-mode network with two non-empty vertex sets $U$ and $V$, edge set $E \subseteq U \times V$, and $\sigma: E \rightarrow\{+1,-1\}$ is the sign function. We denote the size of $U$ and $V$ by $n_{1}$ and $n_{2}$, respectively.

The edge set $E$ of a signed (one-mode or two-mode) network can be

\footnotetext{
${ }^{1}$ The presented results can be generalized to disconnected networks by considering each component separately.
} 
partitioned into the set of positive ties $E^{+}$, such that $\sigma(\{j, k\})=+1 \Longleftrightarrow$ $\{j, k\} \in E^{+}$, and the set of negative edges $E^{-}$with $\sigma(\{j, k\})=-1 \Longleftrightarrow$ $\{j, k\} \in E^{-}$.

Besides general graph terminology, we also need some notations for complex numbers $z \in \mathbb{C}$. The complex conjugate of $z=x+y i$ is defined as $z^{*}=x-y i$ and its absolute value is given by $|z|=\sqrt{x^{2}+y^{2}}$. The conjugate transpose of a matrix $Z \in \mathbb{C}^{n, n}$ is denoted by $Z^{H}$ and its entries are defined as $\left(Z^{H}\right)_{j k}=Z_{k j}^{*}$.

\section{Analyzing (signed) two-mode networks}

As for unsigned two-mode networks, there are two ways of analyzing a signed two-mode network: The "direct" and the "conversion" approach (Borgatti and Halgin, 2011). The direct approach dictates that both modes need to be treated equally and analyzed simultaneously. Since the data is not tampered with, there is no need to fear the loss of information. However, the approach may require methods and algorithms that are tailored to bipartite graphs and standard network analytic tools are not directly applicable. The direct approach has thus far been the preferred one for signed networks, mostly in conjunction with blockmodeling techniques (Doreian et al., 2013; Doreian and Mrvar, 2009).

The conversion approach includes all methods that project the two-mode network onto each mode separately. Projections are defined in terms of the $n_{1} \times n_{2}$ adjacency matrix $A$ of a two-mode network $G=(U, V, E)$. The adjacency matrix of the row and column projections are given by $A A^{T}$ and $A^{T} A$, respectively.

The most obvious application of projections is if only one mode is of interest (the primary mode), but measuring the relationships directly is not 
feasible. The indirect relations via the secondary mode then serves as an indicator for relationships among the nodes in the primary mode. The conversion approach is usually criticized for potentially losing structural properties inherent to the original two-mode network. Everett and Borgatti (2013) argue, though, that this can be mitigated by looking at both projections simultaneously.

While projections constructed via matrix multiplication result in familiar one-mode networks, they usually end up being a fairly dense weighted network, which pose many challenges by itself. For this reason, different methods have been developed to dichotomize the network, sometimes referred to as backbone extraction of the underlying weighted network. The most straightforward way of dichotomizing a weighted network is by fixing a threshold $\varepsilon$ and only include an edge if its weight exceeds $\varepsilon$. Neal (2014) argues that using such an unconditional threshold has a high degree of arbitrariness (how to choose $\varepsilon$ ?), suffers from structural biases (different choices of $\varepsilon$ can yield networks with unpredictably different structural properties), and uniscalarity. The author introduces the stochastic degree sequence model (SDSM) as an alternative method, which allows to condition the threshold on the degree of the underlying two-mode network. The model is defined as follows (exemplified with the row mode). As a first step, a binary outcome model is fitted which predicts the entries $A_{j k}$ of the adjacency matrix associated with the two-mode network based on the row and column sums of $A$. The coefficients are then used to estimate the probabilities $p_{j k}$ of a row node $j$ connecting with a column node $k$. Then, $N$ random two-mode networks $A^{\prime}$ are created where $A_{j k}^{\prime}$ is the outcome of one Bernoulli trial with probability $p_{j k}$, and its projection $B^{\prime}=A^{\prime} A^{\prime T}$ is constructed. A tie between two row nodes $j$ and $l$ is included in the dichotomized one-mode projection 
if $B_{j l}^{\prime}>\left(A A^{T}\right)_{j l}$ in less then $\alpha N$ projections of sampled matrices, where $\alpha$ is the desired significance level. The extracted backbone therefore only includes an edge, if the weight significantly exceeds random chance in the SDSM. For more technical details we refer to Neal (2014).

The SDSM, however, is not the only available method to dichotomize one-mode projections. Others include the fixed degree sequence model (Horvát and Zweig, 2013; Zweig and Kaufmann, 2011) or the disparsity filter (Serrano et al., 2009). All methods come with their own set of advantages and disadvantages and mainly differ in the definition of an underlying Null model. In the absence of a proper validation method, though, there is no way to determine which method "works best". Hence, the goal of the upcoming part is to make any of the proposed dichotomization methods available for signed two-mode networks.

\section{Projecting signed two-mode networks}

We discuss two different projection approaches for signed two-mode networks, a straightforward approach based on matrix multiplication and a method relying on vertex duplication. To the best of our knowledge, the only application of projections so far can be found in Doreian et al. (2013). The authors use the signed adjacency matrix $A$ and consider the net weights of $A A^{T}$ dichotomized by setting a global threshold $\varepsilon$.

\subsection{Matrix multiplication}

The usual way of constructing one-mode projections is via the matrix products $A A^{T}$ and $A^{T} A$. To illustrate why using these direct projections may produce undesirable results, consider the example signed two-mode network shown in Figure 1. 


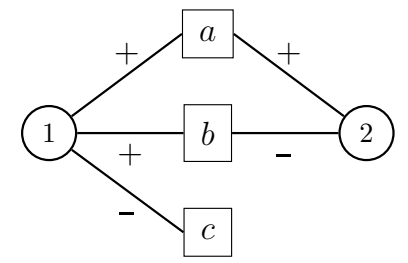

Figure 1: Example of a signed two-mode network.

Its signed adjacency matrix $A$ is given by

$$
A=\left(\begin{array}{rrr}
1 & 1 & -1 \\
1 & -1 & 0
\end{array}\right)
$$

and for the two projections, we obtain

$$
A A^{T}=\left(\begin{array}{ll}
3 & 0 \\
0 & 2
\end{array}\right) \text { and } A^{T} A=\left(\begin{array}{rrr}
2 & 0 & -1 \\
0 & 2 & -1 \\
-1 & -1 & 1
\end{array}\right) \text {. }
$$

Note how negative and positive relations nullify each other, suggesting that there is no relation between node 1 and 2 or $a$ and $b$. To circumvent this problem, we propose to split the signed adjacency matrix into two matrices $A^{+}$and $A^{-}$which hold the positive and negative ties, respectively. That is, $A_{j k}^{+}=1$ if $\{j, k\} \in E^{+}$and $A_{j k}^{-}=1$ if $\{j, k\} \in E^{-}$. This allows us to define two distinct projections per mode. The positive and negative projections for the row mode are defined as

$$
P=A^{+} A^{+^{T}}+A^{-} A^{-T} \text { and } N=A^{+} A^{-T} .
$$

The projections of the column mode can be obtained in the same manner. The projections make use of the natural multiplication rules. e.g. two 
negative edges become one positive. ${ }^{2}$ For the row mode in the example, we obtain

$$
P=\left(\begin{array}{cc}
3 & 1 \\
1 & 2
\end{array}\right) \text { and } N=\left(\begin{array}{cc}
0 & 1 \\
1 & 0
\end{array}\right) .
$$

The diagonal of $P$ contains the unsigned degree in the two-mode network and the diagonal entries of $N$ are always zero. The off-diagonal entries in $P$ count the number of times that two row nodes connect to a column node with the same sentiment, whereas the entries in $N$ count the number of times they connect with differing sentiment. The matrices define two weighted networks, one with positive and one with negative ties only, which can be dichotomized separately. For illustrative purposes, we dichotomize $P$ and $N$ with a threshold of $\varepsilon=1$ such that we obtain adjacency matrices ${ }^{3}$

$$
A^{(P)}=\left(\begin{array}{ll}
0 & 1 \\
1 & 0
\end{array}\right) \text { and } A^{(N)}=\left(\begin{array}{ll}
0 & 1 \\
1 & 0
\end{array}\right) \text {. }
$$

The resulting unweighted networks could now be analyzed separately. For the positive network, we have all common network analytic tools at disposal. Some methods for networks with only negative ties were suggested by Everett and Borgatti (2014). This approach, however, clearly misses the interaction between positive and negative ties. Combining the two networks into one signed network, though, needs to be done with care. Intuitively, $A^{(P)}-A^{(N)}$ should suffice. However, this again nullifies some relations (cf. matrices in Equation (5)). The question is, if we can combine positive and negative ties in a meaningful way to form a new type of relation.

Abelson and Rosenberg (1958) introduced a logic that includes four re-

\footnotetext{
${ }^{2}$ Note that this also implies an underlying model of structural balance.

${ }^{3}$ diagonal entries are set to zero by default to avoid loops.
} 
lations between cognitive elements: positive $(p)$, negative $(n)$, null $(0)$, and ambivalent $(a)$. The element of interest here is the ambivalent relation, which the authors define as "conjunctions of positive and negative relations" that are "psychologically secondary or derived". Given the set of possible values $S=\{p, n, 0, a\}$, the authors introduce addition (" $\oplus$ ") and multiplication ("๑") rules on $S$, shown in Table 1 . It is easy to verify that $(S, \oplus, \odot)$ then forms a semiring (Batagelj, 1994).

Table 1: Addition and multiplication table for $\oplus$ and $\odot$.

\begin{tabular}{c|ccccc|cccc}
$\oplus$ & $p$ & $n$ & 0 & $a$ & $\odot$ & $p$ & $n$ & 0 & $a$ \\
\hline$p$ & $p$ & $a$ & $p$ & $a$ & $p$ & $p$ & $n$ & 0 & $a$ \\
$n$ & $a$ & $n$ & $n$ & $a$ & $n$ & $n$ & $p$ & 0 & $a$ \\
0 & $p$ & $n$ & 0 & $a$ & 0 & 0 & 0 & 0 & 0 \\
$a$ & $a$ & $a$ & $a$ & $a$ & $a$ & $a$ & $a$ & 0 & $a$
\end{tabular}

We can employ the addition rule in our context by replacing all ones in $A^{(P)}$ with $p$ and all ones in $A^{(N)}$ with $n$ and then compute the sum $A^{(P)} \oplus A^{(N)}$.

For the example we thus finally obtain

$$
A^{(P)} \oplus A^{(N)}=\left(\begin{array}{ll}
0 & p \\
p & 0
\end{array}\right) \oplus\left(\begin{array}{ll}
0 & n \\
n & 0
\end{array}\right)=\left(\begin{array}{ll}
0 & a \\
a & 0
\end{array}\right) .
$$

The complete projection approach is summarized in Table 2 .

Table 2: Steps of the matrix multiplication approach (row mode).

a) Define the positive and negative adjacency matrices $A^{+}$and $A^{-}$.

b) Calculate $P=A^{+} A^{+^{T}}+A^{-} A^{-T}$ and $N=A^{+} A^{-T}$.

c) Dichotomize $P$ and $N$ to obtain $A^{(P)}$ and $A^{(N)}$.

d) Compute $A^{(P)} \oplus A^{(N)}$ with Table 1 .

The disadvantage of the matrix multiplication approach is that we have to deal with two separate matrices. Furthermore, not all binarization meth- 
ods are applicable. The SDSM, for instance, requires to fit a binary outcome model on the initial two-mode network. For signed networks, this would require a multinomial approach to compute three tie probabilities (null, positive and negative) and a more complex sampling scheme of random two-mode networks.

\subsection{Vertex duplication approach}

In this section, we introduce a projection method that directly computes the signed one-mode projection, incorporating the possibility of ambivalent ties, without requiring several matrices. The approach is illustrated by fixing a primary mode, but we note that it is also applicable for the secondary mode.

The idea of the approach is to transform the signed two-mode network into an unsigned version, allowing to choose any of the available methods to dichotomize the network. This transformation is done using a vertex $d u$ plication rule. The nodes of the primary mode are duplicated and equipped with a positive and negative sign. Nodes of the secondary mode remain unchanged and connect to the signed nodes according to the signed ties in the original signed two-mode network. More formally, assuming that $U$ is the primary mode, we construct a two-mode network $G^{\star}=\left(U^{\star}, V, E^{\star}\right)$ where $U^{\star}=\left\{u_{1}^{+}, u_{1}^{-}, u_{2}^{+}, u_{2}^{-}, \ldots, u_{n_{1}}^{+}, u_{n_{1}}^{-}\right\}$and

$$
\begin{aligned}
& \left\{u_{j}, v_{k}\right\} \in E^{+} \Longleftrightarrow\left\{u_{j}^{+}, v_{k}\right\} \in E^{\star} \text { and } \\
& \left\{u_{j}, v_{k}\right\} \in E^{-} \Longleftrightarrow\left\{u_{j}^{-}, v_{k}\right\} \in E^{\star}
\end{aligned}
$$

Figure 2 illustrates the duplication rule with a simple example.

The network is now a standard two-mode network so that any projection method can be applied without modification. Once the network is projected and dichotomized, we need to turn it back into a signed network using a 


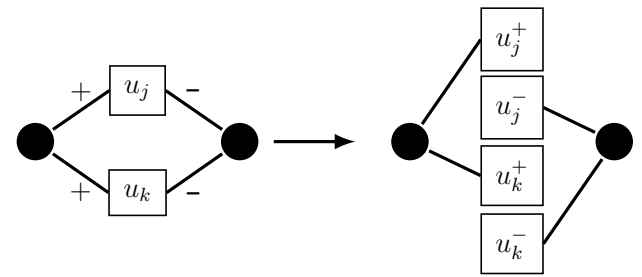

Figure 2: Illustration of the vertex duplication rule to turn a signed twomode network into an unsigned network.

vertex contraction rule. Let $H^{\star}=\left(U^{\star}, E_{H}^{\star}\right)$ be the resulting dichotomized one-mode projection of $G^{\star}=\left(U^{\star}, V, E^{\star}\right)$. Construct $H=\left(U, E_{H}, \sigma\right)$ with $\sigma: E_{H} \rightarrow\{p, n, a\}$ such that

$\left\{u_{j}, u_{k}\right\} \in E_{H}$ and $\sigma\left(\left\{u_{j}, u_{k}\right\}\right)=p$

if $\left\{u_{j}^{+}, u_{k}^{+}\right\} \in E_{H}^{\star}$ or $\left\{u_{j}^{-}, u_{k}^{-}\right\} \in E_{H}^{\star}$ and $\left\{u_{j}^{-}, u_{k}^{+}\right\},\left\{u_{j}^{+}, u_{k}^{-}\right\} \notin E_{H}^{\star}$

$\left\{u_{j}, u_{k}\right\} \in E_{H}$ and $\sigma\left(\left\{u_{j}, u_{k}\right\}\right)=n$

if $\left\{u_{j}^{-}, u_{k}^{+}\right\} \in E_{H}^{\star}$ or $\left\{u_{j}^{+}, u_{k}^{-}\right\} \in E_{H}^{\star}$ and $\left\{u_{j}^{+}, u_{k}^{+}\right\},\left\{u_{j}^{-}, u_{k}^{-}\right\} \notin E_{H}^{\star}$

$\left\{u_{j}, u_{k}\right\} \in E_{H}$ and $\sigma\left(\left\{u_{j}, u_{k}\right\}\right)=a$

if $\left\{u_{j}^{-}, u_{k}^{+}\right\} \in E_{H}^{\star}$ or $\left\{u_{j}^{+}, u_{k}^{-}\right\} \in E_{H}^{\star}$ and $\left\{u_{j}^{+}, u_{k}^{+}\right\} \in E_{H}^{\star}$ or $\left\{u_{j}^{-}, u_{k}^{-}\right\} \in E_{H}^{\star}$.

An illustration of the vertex contraction rules is given in Figure 3 and the complete approach is summarized in Table 3.

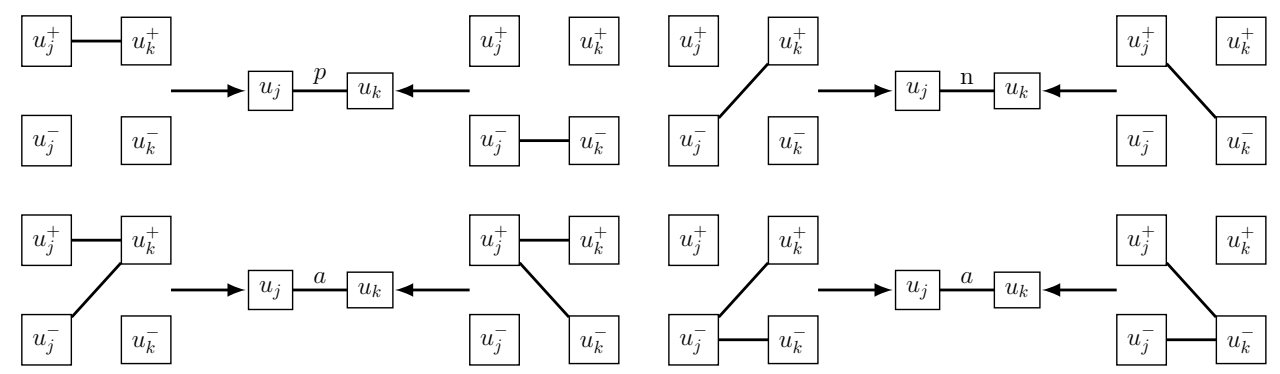

Figure 3: Illustration of the vertex contraction rules. 
Table 3: Steps of the vertex duplication approach.

a) Convert the signed two-mode network into an unsigned network using the vertex duplication rule.

b) Project and dichotomize the unsigned two-mode network

c) Use the vertex contraction rule to obtain the signed projection.

The advantage of vertex duplication over matrix multiplication is that, after duplication, we are dealing with a standard two-mode network. Hence, we do not have to be concerned with any particularities of signed ties in order to project the network.

\section{Analysing projections with ambivalent ties}

Independent of the chosen projection method, the resulting dichotomized one-mode projection may contain ambivalent ties. This not only posses conceptual challenges, but also technical ones. To overcome the technical difficulties, we propose to employ complex versions of the adjacency and Laplacian matrix. This allows to examine the conceptual challenges, such as implications for balance theory, with a sound mathematical basis.

\subsection{Adjacency and Laplacian matrices}

Many network analytic tools are derived from the adjacency matrix of the network. Eigenvector centrality, for instance, is defined as the dominant eigenvector of the adjacency matrix (Bonacich, 1972). The concept has been adapted to signed networks by Bonacich and Lloyd (2004) using $A \in \mathbb{R}^{n, n}$ with entries

$$
A_{j k}= \begin{cases}\sigma(\{j, k\}) & \{j, k\} \in E \\ 0 & \text { else, }\end{cases}
$$


where $\sigma: E \rightarrow\{+1,-1\}$. Ambivalent ties, however, do not fit into this scheme in any natural way. Intuitively, they should be placed somewhere between positive and negative ties, yet this value is obviously taken by the null tie. Using the symbolic logic by Abelson and Rosenberg (1958) introduced in Section 4 is one possibility to deal with this issue. Batagelj (1994), for instance, used the induced semiring for the structural balance problem. However, the semiring is too restrictive and impractical for other empirically relevant tasks, such as deriving an eigendecomposition. This is why we turn to the work of Cartwright and Harary (1970), who introduced a related but technically more approachable notion for signed networks including ambivalent ties. The authors present a framework where edges $e=(i, j)$ are encoded with $m(e)=(\rho, \eta)$ where $\rho, \eta \in[0,1]$ indicate the positive and negative part of the tie, respectively. Purely positive and negative ties are defined as $m(e)=(1,0)$ and $m(e)=(0,1)$, and ambivalent ties as $m(e)=(0.5,0.5) \cdot{ }^{4}$ Note that $(1,0) \cong p,(0,1) \cong n$ and $(0.5,0.5) \cong a$ such that we can easily switch between symbolic logic and the framework of Cartwright and Harary. Using the fact that $m(e)=(\rho, \eta)$ can be written in terms of complex numbers as $\rho+\eta i$, we can define a new adjacency matrix as follows.

Definition 1. The complex adjacency matrix $\mathcal{A} \in \mathbb{C}^{n, n}$ of an undirected signed network $\Gamma=(G, \sigma)$ with $\sigma: E \rightarrow\{p, n, a\}$ is a Hermitian matrix

\footnotetext{
${ }^{4}$ The authors define two models with different representations for ambivalent ties. the diamond model with $(1,1)$ and the triangle model with $(0.5,0.5)$. The triangle model is chosen here since the positive and negative parts sum up to one.
} 
defined as

$$
\mathcal{A}_{j k}= \begin{cases}1+0 i & \sigma(\{j, k\})=p \\ 0+1 i & \sigma(\{j, k\})=n \\ 0.5+0.5 i & \sigma(\{j, k\})=a \\ 0+0 i & \text { else. }\end{cases}
$$

if $j \leq k$ and

$$
\mathcal{A}_{k j}=\mathcal{A}_{j k}^{*}
$$

for $j>k$.

The Hermitian property is not absolutely essential. Yet it supplies the adjacency matrix with some convenient properties, e.g. real eigenvalues. To the best of our knowledge, Hermitian matrices have so far only been considered for directed (Cucuringu et al., 2019; Guo and Mohar, 2017) or mixed graphs (Mohar, 2016; Yu and Qu, 2015).

With the definition of the complex adjacency matrix, it is straightforward to define the complex Laplacian matrix.

Definition 2. The complex Laplacian matrix $\mathcal{L} \in \mathbb{C}^{n, n}$ is defined as

$$
\mathcal{L}=\mathcal{D}-\mathcal{A},
$$

where $\mathcal{D}$ is a diagonal matrix with entries

$$
\mathcal{D}_{j j}=\sum_{k=1}^{n}\left|\mathcal{A}_{j k}\right|
$$

The normalized complex Laplacian matrix is given by

$$
\mathcal{L}^{\text {norm }}=\mathcal{D}^{-\frac{1}{2}} \mathcal{L D}^{-\frac{1}{2}}=I-\mathcal{D}^{-\frac{1}{2}} \mathcal{A} \mathcal{D}^{-\frac{1}{2}},
$$


where $I$ is the identity matrix.

The following theorem shows that the complex Laplacian matrix inherits an important property from real-valued Laplacian matrices.

Theorem 1. The complex Laplacian matrix $\mathcal{L}$ of a signed network is positive semi-definite.

Proof. Let $\Gamma=(G, \sigma)$ be an undirected signed network and $\mathcal{A}$ its complex adjacency matrix. Orient each edge $(j, k)$ such that $j<k$. Let $\mathcal{S} \in \mathbb{C}^{n, m}$ be the incidence matrix of $\Gamma$ with entries

$$
\begin{aligned}
\mathcal{S}_{j(j, k)} & =\sqrt{\mathcal{A}_{j k}} \\
\mathcal{S}_{k(j, k)} & = \begin{cases}-\sqrt{A_{k j}} & \mathcal{A}_{j k}=0.5+0.5 i \\
-\mathcal{A}_{k j} \sqrt{\mathcal{A}_{k j}} & \text { else. }\end{cases}
\end{aligned}
$$

It follows that

$$
\begin{aligned}
& \left(\mathcal{S} \mathcal{S}^{H}\right)_{j j}=\sum_{(j, k) \in E} \sqrt{\mathcal{A}_{j k}} \sqrt{\mathcal{A}_{j k}^{*}}=\sum_{(j, k) \in E}\left|\mathcal{A}_{j k}\right| \\
& \left(\mathcal{S} \mathcal{S}^{H}\right)_{j k}=-\mathcal{A}_{j k} \\
& \left(\mathcal{S} \mathcal{S}^{H}\right)_{k j}=-\mathcal{A}_{j k}^{*}=-\mathcal{A}_{k j},
\end{aligned}
$$

Therefore,

$$
\mathcal{L}=\mathcal{S S}^{H}
$$

which implies that $\mathcal{L}$ is positive semi-definite.

\subsection{Structural balance and ambivalent ties}

The concept of balance was introduced by Heider (1946) and later adopted for signed networks by Cartwright and Harary (1956). The basic properties of a balanced signed network are summarized in the following theorem. 
Theorem 2 (Cartwright and Harary (1970)). The following statements are equivalent for a symmetric signed network $\Gamma=(G, \sigma)$, where $\sigma: E \rightarrow$ $\{+1,-1\}$.

(i) The value of every cycle is +1 .

(ii) For every pair of vertices, the values of all paths joining them are the same.

(iii) The set $V$ can be partitioned into two subsets (one of which may be empty) such that the value of each tie between two vertices of the same set is +1 and the value of each tie between two vertices of different subsets is -1 .

The implications for Theorem 2 in the presents of ambivalent ties were explored by Cartwright and Harary (1970). The cycle and partition criterion can obviously not be satisfied if the network contains any ambivalent tie. The authors offer a new version of the path criterion including ambivalent ties (see Cartwright and Harary (1970) Theorem 5) with the consequence that the three statements of Theorem 2 are no longer equivalent. We can thus distinguish between cycle balanced, path balanced, and partition balanced networks. Note that ambivalent ties are absorbing for paths (and cycles). That is, the value of a path is always $a$ as soon as one ambivalent tie is present. It is therefore virtually impossible to formally extend path and cycle balance in any meaningful way for ambivalent ties. For partition balance, however, there is a wider scope. The following definition extends the concept to include a version for networks containing ambivalent ties.

Definition 3. A symmetric signed network $\Gamma=(G, \sigma)$, where $\sigma: E \rightarrow$ $\{p, n, a\}$ is 
(i) strongly partition balanced if $V$ can be partitioned into two subsets (one of which may be empty) such that the value of each tie between two vertices of the same set is $p$ and the value of each tie between two vertices of different subsets is $n$.

(ii) weakly partition balanced if $V$ can be partitioned into two subsets (one of which may be empty) such that the value of each tie between two vertices of the same set is $p$ and the value of each tie between two vertices of different subsets is a.

(iii) partition unbalanced if it is neither strongly nor weakly partition balanced.

Evidently, strongly partition balanced networks can only contain positive and negative ties and corresponds to partition balance as given in Theorem 2 (iii). A strongly partition balanced network is therefore also cycle balanced. Weakly partition balanced networks are composed of only positive and ambivalent ties and can not be cycle balanced. A network is partition unbalanced as soon as it contains both negative and ambivalent ties.

Kunegis et al. (2010) showed that the signed Laplacian matrix $\bar{L}$ is positive-definite if and only if the network is unbalanced. ${ }^{5}$ In the following, we extend this result to complex Laplacian matrices and the newly introduced definitions of strong and weak partition balance.

Lemma 1. The complex Laplacian matrix of a partition unbalanced network is positive definite.

Proof. Let $\Gamma=(G, \sigma)$ be a signed network with $\sigma: E \rightarrow\{p, n, a\}$ and $\mathcal{L} \in \mathbb{C}^{n, n}$ its complex Laplacian matrix. We show that if $z^{H} \mathcal{L} z=0$ for some

\footnotetext{
${ }^{5}$ This was already observed by Hou et al. (2003).
} 
$z \neq 0$, then there exists a bipartition of vertices which complies either with strong or weak partition balance. Define

$$
\mathcal{L}=\sum_{\{j, k\} \in E} \mathcal{L}^{(j, k)}
$$

where $\mathcal{L}^{(j, k)} \in \mathbb{C}^{n, n}$ contains four non-zero entries

$$
\begin{aligned}
& \mathcal{L}_{j j}^{(j, k)}=\mathcal{L}_{k k}^{(j, k)}=\left|\mathcal{A}_{j k}\right| \\
& \mathcal{L}_{j k}^{(j, k)}=-\mathcal{A}_{j k} \\
& \mathcal{L}_{k j}^{(j, k)}=-\mathcal{A}_{j k}^{*},
\end{aligned}
$$

assuming that $j<k$. Let $z \in \mathbb{C}^{n}$ be a non-zero vector with entries $z_{j}=$ $x_{j}+y_{j} i$. Then

$$
\begin{aligned}
z^{H} \mathcal{L}^{(j, k)} z & =\left|\mathcal{A}_{j k}\right|\left(x_{j}^{2}+y_{j}^{2}\right)-\mathcal{A}_{j k}^{*}\left(x_{j} x_{k}+y_{j} y_{k}+\left(x_{k} y_{j}-y_{k} x_{j}\right) i\right) \\
& -\mathcal{A}_{j k}\left(x_{j} x_{k}+y_{j} y_{k}-\left(x_{k} y_{j}-y_{k} x_{j}\right) i\right)+\left|\mathcal{A}_{j k}\right|\left(x_{k}^{2}+y_{k}^{2}\right)
\end{aligned}
$$

We first assume that there only exist positive and negative ties. If $\mathcal{A}_{j k}=$ $1+0 i$ we have

$$
\begin{aligned}
z^{H} \mathcal{L}^{(j, k)} z & =x_{j}^{2}+y_{j}^{2}+x_{k}^{2}+y_{k}^{2}-2\left(x_{j} x_{k}+y_{j} y_{k}\right) \\
& =\left(x_{j}-x_{k}\right)^{2}+\left(y_{j}-y_{k}\right)^{2}
\end{aligned}
$$

and thus

$$
z^{H} \mathcal{L}^{(j, k)} z=0 \Longleftrightarrow x_{j}=x_{k} \text { and } y_{j}=y_{k}
$$

For $\mathcal{A}_{j k}=0+1 i$, we have

$$
\begin{aligned}
z^{H} \mathcal{L}^{(j, k)} z & =x_{j}^{2}+y_{j}^{2}+x_{k}^{2}+y_{k}^{2}+2\left(x_{j} y_{k}-x_{k} y_{j}\right) \\
& =\left(x_{j}+y_{k}\right)^{2}+\left(x_{k}-y_{j}\right)^{2} .
\end{aligned}
$$


Hence,

$$
z^{H} \mathcal{L}^{(j, k)} z=0 \Longleftrightarrow x_{j}=-y_{k} \text { and } x_{k}=y_{j}
$$

Since $G$ is connected, it follows that $\left|z_{j}\right|=c$ for all $j \in V$. W.l.o.g. assume that $\left|z_{j}\right|=1$. Then, we can define a bijection from the vertex set to $z_{1}=$ $1+0 i$ and $z_{2}=0+1 i$, which defines a bisection of the vertices as postulated in Theorem 2.

Assume now, that there only exist positive and ambivalent ties. For $\mathcal{A}_{j k}=0.5+0.5 i$, we obtain

$$
z^{H} \mathcal{L}^{(j, k)} z=\sqrt{1 / 2}\left(x_{j}^{2}+y_{j}^{2}+x_{k}^{2}+y_{k}^{2}\right)-x_{j} x_{k}-y_{j} y_{k}+x_{j} y_{k}-x_{k} y_{j}
$$

A possible solution is given by

$$
y_{j}=0, x_{k}=\frac{x_{j}}{\sqrt{2}} \text { and } y_{k}=-\frac{x_{j}}{\sqrt{2}} \Longrightarrow z^{H} \mathcal{L}^{(i, j)} z=0 \text {. }
$$

With a similar reasoning as above, we define a bijection from the vertex set to $z_{1}=1+0 i$ and $z_{2}=\frac{1}{\sqrt{2}}-\frac{1}{\sqrt{2}} i$, which defines a weakly partition balanced bisection of the vertex set.

If all types of ties are present, then Equation (24) and Equation (26) together imply $z_{j}=0$ for all $j \in V$. Hence, $z^{H} \mathcal{L} z=0$ only holds if $G$ is strongly or weakly partition balanced.

Next, we show that the eigendecomposition of the complex Laplacian matrix of a strongly partition balanced network $\Gamma=(G, \sigma)$ is related to the eigendecomposition of the Laplacian matrix of the underlying unsigned network $G$.

Lemma 2. Let $\Gamma=(G, \sigma)$ be a strongly partition balanced network, and let $z$ 
be the partition vector with entries $z_{j} \in\{1+0 i, 0+1 i\}$ for all $j \in V$. Let $\mathcal{L}$ be the complex Laplacian matrix and $\mathcal{L}=U \Lambda U^{H}$ its eigendecomposition. The eigendecomposition of the Laplacian matrix $L$ of the underlying unsigned network $G$ is given by $L=X \Lambda X^{T}$ where $X_{j k}=z_{j} U_{j k}$.

Proof. To show that $L=X \Lambda X^{T}$, note that

$$
X_{j .} \Lambda X_{j .}^{T}=z_{j} U_{j} . \Lambda z_{j}^{*} U_{j}^{H}=\left|z_{j}\right|^{2} U_{j} . \Lambda U_{j .}^{H}=\mathcal{L}_{j j}=L_{j j}
$$

and

$$
X_{j} . \Lambda X_{k}^{T}=z_{j} U_{j} . \Lambda z_{k}^{*} U_{k}^{H}=z_{j} z_{k}^{*} \mathcal{L}_{j k}=L_{j k},
$$

where the last equality can be checked with basic complex arithmetics. Additionally, it holds that

$$
X_{\cdot k}^{T} X_{\cdot l}=\sum_{j} z_{j}^{*} z_{j} U_{j k}^{H} U_{j l}=0
$$

for $k \neq l$ since $z_{j}^{*} z_{j}=1$ for $z_{j} \in\{1+0 i, 0+1 i\}$. Hence, $X$ is an orthogonal matrix and $L=X \Lambda X^{T}$ is a proper eigendecomposition.

The case of weakly partition balanced networks becomes more involved, since the underlying unsigned network can no longer be constructed straightforwardly.

Definition 4. Let $\Gamma=(G, \sigma)$ be a weakly partition balanced network. The underlying unsigned network $G=(V, E, \omega)$ is a weighted network where for $\{j, k\} \in E$, it holds that

$$
\omega(\{j, k\})= \begin{cases}1 & \sigma(\{j, k\})=p \\ \sqrt{\frac{1}{2}} & \sigma(\{j, k\})=a\end{cases}
$$


Lemma 3. Let $\Gamma=(G, \sigma)$ be a weakly partition balanced network and let $z$ be the partition vector with entries $z_{j} \in\left\{1+0 i, \frac{1}{\sqrt{2}}-\frac{1}{\sqrt{2}} i\right\}$ for all $j \in V$. Let $\mathcal{L}$ be the complex Laplacian matrix and $\mathcal{L}=U \Lambda U^{H}$ its eigendecomposition. The eigendecomposition of the Laplacian matrix $L$ of the underlying weighted unsigned network $G$ is given by $L=X \Lambda X^{T}$ where $X_{j k}=z_{j} U_{j k}$.

Proof. Analogous to Lemma 2.

We can now state the main result.

Theorem 3. The complex Laplacian matrix of a signed network $\Gamma=(G, \sigma)$ with $\sigma: E \rightarrow\{p, n, a\}$ is positive definite if and only if the network is partition unbalanced.

Proof. follows directly from Lemmas 1 to 3.

The theorem provides a simple method to check for strong and weak partition balance and identify the partitioning into two groups. A network is partition balanced if if the complex Laplacian matrix is positive semidefinite. It just remains to check if all non-positive ties are either negative or ambivalent to establish strong or weak balance. The respective partitioning can be constructed from the eigenvector corresponding to the null eigenvalue. In the case of strong partition balance, all entries are either $x+0 i$ or $0-x i$. The entries in the case of weak partition balance are given by $x+0 i$ and $\sqrt{x}+\sqrt{x} i$.

\section{Conclusion}

We presented two methods to project signed two-mode networks that allow the application of known dichotomization tools for unsigned two-mode networks. The aim of these tools is to turn weighted projections into more 
manageable unweighted networks where only the most significant ties are included. In the case of signed two-mode networks, however, we showed that dichotomized projections are not necessarily pure signed networks but may include ambivalent ties. Ambivalence in the context of signed networks was introduced by Abelson and Rosenberg (1958), but has not received much consideration since then. This may partially be due to measurement issues. It is not immediately evident how an ambivalent relation between actors or entities can directly be measured or inferred. This issue is mitigated in our case since ambivalent ties arise indirectly from the projections. Clearly more rigorous empirical work is necessary to establish the relevance and interpretability of ambivalent ties in general, not only for those derived from projections. So far, only some hypothetical results exist. Doreian (2017) suggests that ambivalence "could point to the temporal instability of ties that could be positive at one point in time but negative at another". The reasoning of Cartwright and Harary (1970) relies on balance theory. The authors state that "attitudes of ambivalence should be unstable, changing to positive or negative attitudes so as to satisfy the three criteria [cf. Theorem 2] for balance." Ambivalence does not naturally fit into balance theory and the equivalence of the cycle, path and partition criteria break down as soon as an ambivalent tie is present. We introduced a weaker variant of the partition criterion to accommodate for ambivalence but its empirical relevance remains open.

The presence of ambivalent ties does not only pose conceptual but also technical challenges. To define common matrices associated with networks, we needed to switch from real to complex matrices. This may appear like a technical leap, but it provides new opportunities to adapt network analytic tools for signed networks in the presence of ambivalence. More investiga- 
tions are certainly necessary to establish if complex matrices constituted an appropriate technical foundation. Our initial results are promising since the complex Laplacian matrix inherits some desirable features of its signed version. The next important step is to investigate properties of the complex adjacency matrix and adapt known methods, e.g. based on the eigendecomposition. Note that this already poses some issues for the signed adjacency matrix. Bonacich and Lloyd (2004) introduced eigenvector centrality for signed networks, but ignored the fact that a dominant eigenvalue may not exist (Everett and Borgatti, 2014). Hence, it is not necessarily clear which (combination of) eigenvector(s) to choose as centrality scores. It appears that a non-negligible amount of signed networks have a symmetric spectrum and hence no dominant eigenvector (Akbaria et al., 2018). Some preliminary experiments suggest that the spectrum of the complex adjacency matrix is not necessarily symmetric if the spectrum of the signed adjacency matrix is, and vice versa. However, it remains to show which properties cause symmetric spectra in both cases. Depending on the properties of the complex adjacency matrix, it may well provide a viable alternative to the signed version, even in the absence of ambivalence.

\section{References}

Abelson, R. P. and Rosenberg, M. J. (1958). Symbolic psycho-logic: A model of attitudinal cognition. Behavioral Science, 3(1):1-13.

Akbaria, S., Maimanib, H., and Majdb, L. P. (2018). On the spectrum of some signed complete and complete bipartite graphs. Filomat, $32(17): 5817-5826$. 
Aref, S. and Wilson, M. C. (2018). Measuring partial balance in signed networks. Journal of Complex Networks, 6(4):566-595.

Batagelj, V. (1994). Semirings for social networks analysis. The Journal of Mathematical Sociology, 19(1):53-68.

Bonacich, P. (1972). Factoring and weighting approaches to status scores and clique identification. The Journal of Mathematical Sociology, 2(1):113120.

Bonacich, P. and Lloyd, P. (2004). Calculating status with negative relations. Social Networks, 26(4):331-338.

Borgatti, S. and Halgin, D. (2011). Analyzing affiliation networks. The Sage Handbook of Social Network Analysis, pages 417-433.

Brandes, U., Kenis, P., Lerner, J., and Van Raaij, D. (2009). Network analysis of collaboration structure in Wikipedia. In Proceedings of the 18th International Conference on World Wide Web, pages 731-740. ACM.

Brandes, U. and Lerner, J. (2008). Visual analysis of controversy in usergenerated encyclopedias. Information Visualization, 7(1):34-48.

Cartwright, D. and Harary, F. (1956). Structural balance: A generalization of Heider's theory. Psychological Review, 63(5):277.

Cartwright, D. and Harary, F. (1970). Ambivalence and Indifference in Generalizations of Structural Balance. Behavioral Science, 15(6).

Cucuringu, M., Li, H., Sun, H., and Zanetti, L. (2019). Hermitian matrices for clustering directed graphs: Insights and applications. arXiv:1908.02096 [cs, stat]. 
Davis, J. A. (1967). Clustering and Structural Balance in Graphs. Human Relations, 20(2):181-187.

Doreian, P. (2004). Evolution of human signed networks. Metodoloski Zvezki, $1(2): 277-293$.

Doreian, P. (2008). A multiple indicator approach to blockmodeling signed networks. Social Networks, 30(3):247-258.

Doreian, P. (2017). Reflections on Studying Signed Networks. Journal of Interdisciplinary Methodologies and Issues in Science, Graphs and Social Systems.

Doreian, P., Kapuscinski, R., Krackhardt, D., and Szczypula, J. (1996). A brief history of balance through time. The Journal of Mathematical Sociology, 21(1-2):113-131.

Doreian, P., Lloyd, P., and Mrvar, A. (2013). Partitioning large signed twomode networks: Problems and prospects. Social Networks, 35(2):178-203.

Doreian, P. and Mrvar, A. (1996). A partitioning approach to structural balance. Social Networks, 18(2):149-168.

Doreian, P. and Mrvar, A. (2009). Partitioning signed social networks. Social Networks, 31(1):1-11.

Doreian, P. and Mrvar, A. (2015). Structural balance and signed international relations. Journal of Social Structure, 16:1.

Estrada, E. (2019). Rethinking structural balance in signed social networks. Discrete Applied Mathematics. 
Estrada, E. and Benzi, M. (2014). Walk-based measure of balance in signed networks: Detecting lack of balance in social networks. Physical Review E, 90(4):042802.

Everett, M. G. and Borgatti, S. P. (2013). The dual-projection approach for two-mode networks. Social Networks, 35(2):204-210.

Everett, M. G. and Borgatti, S. P. (2014). Networks containing negative ties. Social Networks, 38:111-120.

Facchetti, G., Iacono, G., and Altafini, C. (2011). Computing global structural balance in large-scale signed social networks. Proceedings of the National Academy of Sciences, 108(52):20953-20958.

Giscard, P.-L., Rochet, P., and Wilson, R. (2017). Evaluating balance on social networks from their simple cycles. Journal of Complex Networks, $5(5): 750-775$.

Guo, K. and Mohar, B. (2017). Hermitian Adjacency Matrix of Digraphs and Mixed Graphs. Journal of Graph Theory, 85(1):217-248.

Heider, F. (1946). Attitudes and Cognitive Organization. The Journal of Psychology, 21(1):107-112.

Horvát, E.-Á. and Zweig, K. A. (2013). A fixed degree sequence model for the one-mode projection of multiplex bipartite graphs. Social Network Analysis and Mining, 3(4):1209-1224.

Hou, Y., Li, J., and Pan, Y. (2003). On the Laplacian Eigenvalues of Signed Graphs. Linear and Multilinear Algebra, 51(1):21-30.

Kamvar, S. D., Schlosser, M. T., and Garcia-Molina, H. (2003). The Eigentrust Algorithm for Reputation Management in P2P Networks. In Pro- 
ceedings of the 12th International Conference on World Wide Web, WWW '03, pages 640-651, New York, NY, USA. ACM.

Kerchove, C. and Dooren, P. (2008). The PageTrust algorithm: How to rank web pages when negative links are allowed? In Proceedings of the 2008 SIAM International Conference on Data Mining, Proceedings, pages 346-352. Society for Industrial and Applied Mathematics.

Kirkley, A., Cantwell, G. T., and Newman, M. E. J. (2019). Balance in signed networks. Physical Review E, 99(1).

Kittur, A., Suh, B., Pendleton, B. A., and Chi, E. H. (2007). He Says, She Says: Conflict and Coordination in Wikipedia. In Proceedings of the SIGCHI Conference on Human Factors in Computing Systems, CHI '07, pages 453-462, New York, NY, USA. ACM.

Kunegis, J., Lommatzsch, A., and Bauckhage, C. (2009). The Slashdot Zoo: Mining a Social Network with Negative Edges. In Proceedings of the 18th International Conference on World Wide Web, WWW '09, pages 741-750, New York, NY, USA. ACM.

Kunegis, J., Schmidt, S., Lommatzsch, A., Lerner, J., De Luca, E., and Albayrak, S. (2010). Spectral Analysis of Signed Graphs for Clustering, Prediction and Visualization. In Proceedings of the 2010 SIAM International Conference on Data Mining, Proceedings, pages 559-570. Society for Industrial and Applied Mathematics.

Leskovec, J., Huttenlocher, D., and Kleinberg, J. (2010). Signed Networks in Social Media. In Proceedings of the SIGCHI Conference on Human Factors in Computing Systems, CHI '10, pages 1361-1370. 
Ma, L., Gong, M., Du, H., Shen, B., and Jiao, L. (2015). A memetic algorithm for computing and transforming structural balance in signed networks. Knowledge-Based Systems, 85:196-209.

Ma, L., Gong, M., Yan, J., Yuan, F., and Du, H. (2017). A decompositionbased multi-objective optimization for simultaneous balance computation and transformation in signed networks. Information Sciences, 378:144160.

Marvel, S. A., Strogatz, S. H., and Kleinberg, J. M. (2009). Energy Landscape of Social Balance. Physical Review Letters, 103(19):198701.

Mohar, B. (2016). Hermitian adjacency spectrum and switching equivalence of mixed graphs. Linear Algebra and its Applications, 489:324-340.

Moore, M. (1979). Structural balance and international relations. European Journal of Social Psychology, 9(3):323-326.

Neal, Z. P. (2014). The backbone of bipartite projections: Inferring relationships from co-authorship, co-sponsorship, co-attendance and other co-behaviors. Social Networks, 39:84-97.

Serrano, M. Á., Boguñá, M., and Vespignani, A. (2009). Extracting the multiscale backbone of complex weighted networks. Proceedings of the National Academy of Sciences, 106(16):6483-6488.

Terzi, E. and Winkler, M. (2011). A Spectral Algorithm for Computing Social Balance. In Frieze, A., Horn, P., and Prałat, P., editors, Algorithms and Models for the Web Graph, Lecture Notes in Computer Science, pages 1-13. Springer Berlin Heidelberg.

Traag, V. A., Nesterov, Y. E., and Van Dooren, P. (2010). Exponential Ranking: Taking into Account Negative Links. In Bolc, L., Makowski, 
M., and Wierzbicki, A., editors, Social Informatics, Lecture Notes in Computer Science, pages 192-202. Springer Berlin Heidelberg.

Wu, Z., Aggarwal, C. C., and Sun, J. (2016). The Troll-Trust Model for Ranking in Signed Networks. In Proceedings of the Ninth ACM International Conference on Web Search and Data Mining, WSDM '16, pages 447-456, New York, NY, USA. ACM.

Yu, G. and Qu, H. (2015). Hermitian Laplacian matrix and positive of mixed graphs. Applied Mathematics and Computation, 269:70-76.

Zweig, K. A. and Kaufmann, M. (2011). A systematic approach to the onemode projection of bipartite graphs. Social Network Analysis and Mining, $1(3): 187-218$. 